\title{
Mensch und Tier in der Kunst
}

Franziska Daniela Burkhardt

\section{«Bei der Arbeit am «trabenden Rentier> fühlte ich \\ plötzlich, wie ich am ganzen Körper zu zucken begann.»}

Korrespondenz:

Franziska Daniela Burkhardt

Obere Gasse 12A

CH-4144 Arlesheim

fburkhardt[at]sunrise.ch
Fleisch und Produkte des Tieres waren für den Menschen notwendig; von gewissen Tieren wurde er bedroht. Das Tier wurde als Kraft, als göttliches Wesen, Geist und Schutz verehrt, angebetet und gefürchtet. Diese komplexe Haltung des Menschen gegenüber dem Tier wird schon seit jeher bildlich dargestellt.

Versteckt in einem Schacht des prähistorischen Höhlenheiligtums Lascaux (Perigord), wird ein stürzender Mann mit Speer und erigiertem Penis gegenüber einem Wisent mit herausquellenden Därmen dargestellt, in der Höhle «Les Trois Frères» (Südfrankreich) überrascht der «Sorcière», halb Mensch, halb Hirsch. Knochen und Geweihe wurden mit Symbolen und Zeichnungen graviert, und Jagdwaffen wurden seit frühesten Zeiten kunstvoll verziert.

Meine ersten Tierbilder kratzte ich unmittelbar in Kupferplatten. Da die Linien kaum sichtbar waren, fand der Hauptprozess nicht über das Auge, sondern über den «Bewegungssinn» statt, eine Körpererinnerung. Haben damals die Künstler aus dieser Körpererinnerung gearbeitet? Oft sind Stellen so gewählt, dass die Abbildungen auf ein natürliches Felsenrelief, das Teile des Tieres bereits wiedergibt, gearbeitet sind so als wären die Tiere auf dem Felsen erspürt worden.
Meine jetzigen Tierbilder entstehen nach längerem Zusammensein mit den Tieren. Ich skizziere, fotografiere und beobachte genau. Ebenfalls studiere ich Skelett und Muskeln. Die Bilder entstehen dann in einem Guss, ohne Entwurf, auf einem flachen, unübersichtlichen Drucktisch. Ich arbeite einzig aus der Erinnerung. Bei der Arbeit am «trabenden Rentier» fühlte ich plötzlich, wie ich am ganzen Körper zu zucken begann. Mir wurde bewusst, dass dies dem Zucken der Rentiere entspricht, mit dem diese sich gegen die Mücken wehren. Am meisten überraschte mich dabei, dass dies durch eine akribische Arbeit im Atelier (Umsetzen der Skizzen, Vermessen der Fotos, Anatomiestudien) geschehen war und nicht im Bann der unmittelbaren Begegnung mit dem Tier. Es scheint, dass dieses genaue Hinsehen und die durch das Tier geweckten Emotionen, die letztlich mehr mit mir und meinen Gefühlen und weniger mit dem Tier selber zu tun haben, zu einer kurzzeitigen Verschmelzung mit dem Tier führen können. Giacometti schildert ein solches Erleben, als er einen Hund modellierte. Die Menschen in der Steinzeit haben die Tiere sehr genau beobachtet, gejagte oder schon tote Tiere ausgeweidet, sie kannten die Tiere sehr genau. Könnte es sein, dass sie beim Darstellen der Tiere quasi zu diesen wurden? Es gibt alte Mythen und Märchen, wo Menschen für eine Zeit als Tiere unter diesen lebten und von ihnen gewisse Eigenschaften erhielten.

Wenn man diese Vermutung weiterverfolgt, würde das bedeuten, dass letztendlich der Moment des Malens, Zeichnens, Gravierens entscheidend und das fertige Bild nur ein Nebenprodukt ist? Die
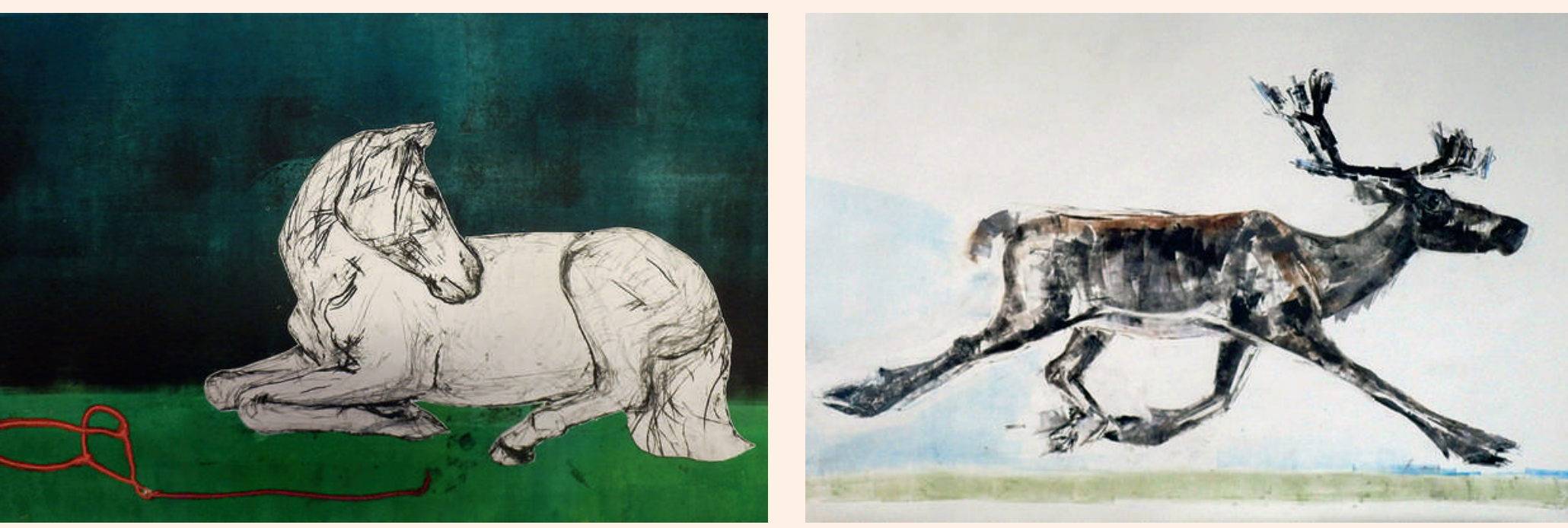

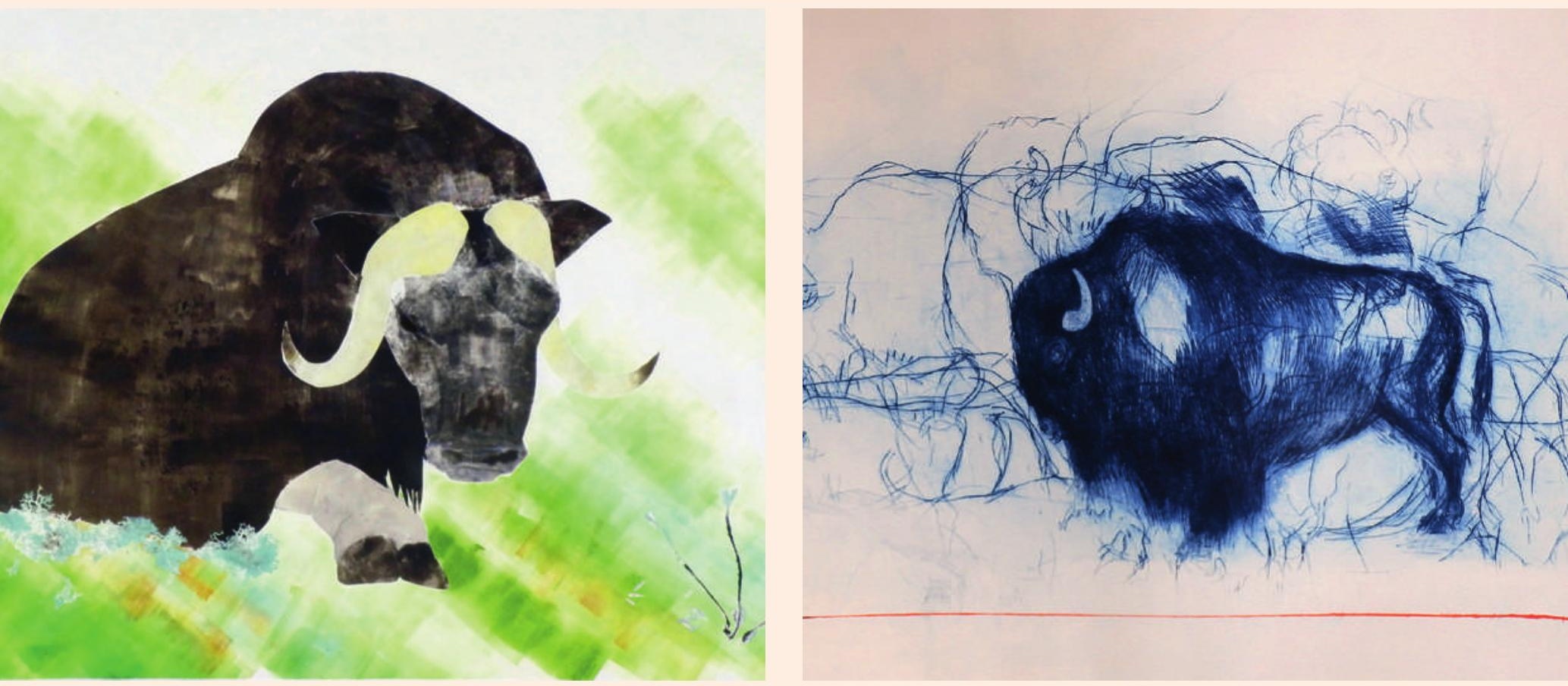

Tatsache, dass die Bilder manchmal an unzugänglichen und schlecht einsehbaren Orten angebracht sind, häufig nicht als Gesamtheit eingesehen werden können, würde die Vermutung verstärken, dass es den Menschen um den Augenblick der Handlung und nicht um das fertige Produkt, um das Sein und nicht das Haben, ging. bronzezeitlichen Messern gemeisselt, von Leuten, die Hunderte von Kilometern reisten, um in diesem Tal ihrem Kult nachzugehen.

In der neueren Zeit haben wir immer mehr das ungetrennte Erleben verloren. Wir begannen, in Religion, Leben, Medizin und Kunst aufzuspalten, und in diesem Prozess wird das Tier in die Mythologie

\section{Der Hauptprozess fand nicht über das Auge, sondern über den «Bewegungssinn» statt, über eine Körpererinnerung.}

Im Laufe der Zeit wurde das Tier durch die Domestikation zum unentbehrlichen Helfer des Menschen. Interessanterweise taucht in Göbekli Tepe, der frühsten bekannten Tempelanlage in der SüdOst-Türkei, die erste mir bekannte geometrisch stilisierte Tierdarstellung auf, ein lebensgrosser Fuchs, etwa im Zeitraum, als der Hund domestiziert wird.

Im Val Camonica in Norditalien gibt es fantastische Gravuren aus der Bronzezeit. Auf offenem Felsen sind wie in einem Bilderbuch Pflüge ziehende Rinder, Pferde vor Wagen, Reiter und zahme Hunde (sie tragen im Unterschied zum wilden Hund den Schwanz nach oben), Jagdszenen, ein Mensch mit Tierkopf gezeichnet. Auch in Südschweden entstehen in dieser Zeit viele in den Felsen gemeisselte Wagendarstellungen. In derselben Zeit werden im hohen Norden Rentierjagden und Fischfang dargestellt.

Im Vallée des Merveilles nördlich von Nizza wurden stilisierte Rinder in rot-erodierten Stein neben eingebaut. In unserer Zeit taucht es in der Kunst auf, wird gegessen, wir brauchen seine Haut und sein Fell, es dient uns als Arbeits- und Freizeitpartner, wird zum Schmusetier, und in der Medizin wird es in der Forschung und in Therapien eingesetzt.

\section{Ausstellung vom 9.-20. April 2013}

\section{Franziska Burkhardt - Bilder und Objekte}

KUNSTpART - Spalenberg 30, 4051 Basel www.kunstpart.ch

Ausstellungsapéro: Dienstag, 9. April, 17-20 Uhr, Finissage: Samstag, 20. April, 15-17 Uhr, mit Anwesenheit der Künstlerin (auch donnerstags 18.30-20 Uhr).

\section{Öffnungszeiten:}

Dienstag-Freitag: 10-12 Uhr und 14-18.30 Uhr, Donnerstag bis $20 \mathrm{Uhr}$

Samstag: 11-17 Uhr. 